\title{
Histone-writer cancer drugs enter center stage
}

\section{Epizyme's EZH2 inhibitor gains two approvals in quick succession - a milestone for this new class of epigenetic cancer drug that targets histone marks with greater precision than previous generations.} Administration (FDA) gave accelerated approval to Epizyme's EZH2 inhibitor Tazverik (tazemetostat) for relapsed or refractory follicular lymphoma, marking the drug's second approval in less than six months; the first was for epithelioid sarcoma, a rare disease until then without green lights in quick succession for this first-in-class EZH2 inhibitor presage more specific epigenetic agents to come for cancer.. Tazverik "is the tip of the iceberg," says Robert Copeland, Epizyme's founding CSO and now president and CSO of Accent Therapeutics.

Tazverik works by inhibiting EZH2, short for enhancer of zeste homolog 2 . It is part of a histone methyltransferase complex, responsible for the trimethylation of lysine 27 on histone $\mathrm{H} 3$. One of its biological effects is to temporarily silence genes involved in the differentiation of $\mathrm{B}$ cells and their exit from the germinal centers in lymph nodes. When the EZH2 gene is mutated, making its function irreversible, B cells are unable to exit and become cancerous, as happens in follicular lymphoma. In a phase 2 trial in relapsed or refractory follicular lymphoma, 31 of 45 patients with mutated EZH2 in their tumors had an objective response to Tazverik, whereas 19 of 54 individuals with non-mutated EZH2 tumors responded. The n 18 June, the US Food and Drug an approved treatment. The agency's two responses in individuals with EZH2-mutant tumors lasted for about a year, and the response rate was higher than for two phosphatidylinositol-3-OH kinase (PI3K) inhibitors previously approved for this indication, with considerably less toxicity. "That [efficacy] speaks to the power of the biology at play," says Ari Melnick, a cancer researcher at the Weill Cornell Medical College. "It's hard to have a strong impact on patients at that stage."

Although the FDA's approval for relapsed or refractory cases was expected, the agency issued a surprisingly expansive label, says Yaron Werber, a biotech analyst at Cowen. It approved the drug for patients with EZH2-mutant lymphomas who have failed two previous therapies, but also for adults "who have no satisfactory alternative treatment options," regardless of mutation status. This gives hematologists wide discretion. Werber forecasts worldwide Tazverik sales peaking at \$959 million in 2032.

Scientists have been developing epigenetic agents for cancer for several decades. Many researchers consider DNA methylation and histone deacetylation just as important as gene mutations in turning off genes, either by blocking transcription factor binding to promoters or by causing chromatin structure to become more compact and inaccessible. But thus far small molecules targeting epigenetic targets haven't met early expectations: they are

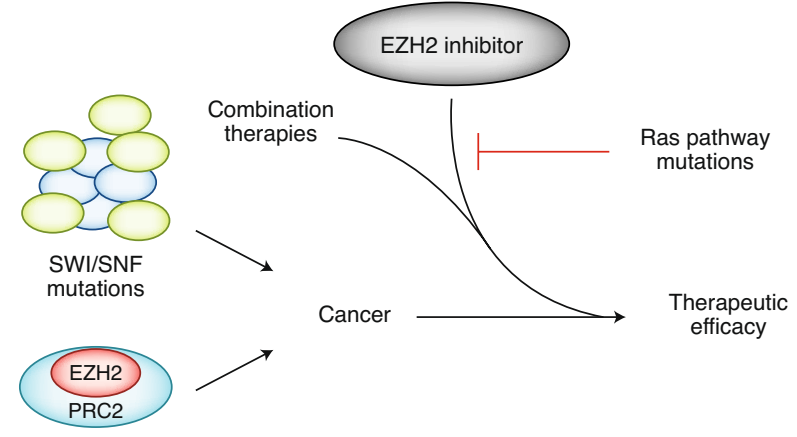

EZH2 gain-of-function mutations (e.g., Y641, A677, A687)

Mutations in either a chromatin remodeling complex or in EZH2 itself can sensitize tumors to EZH2 inhibitors like Tazverik. Reprinted with permission from K. H. Kim and C. W. M. Roberts, Nat. Med. 22, 128-134 (2016).

\section{Antibacterials get \$1 billion injection}

A new public-private fund will invest $\$ 1$ billion to tackle the global problem of antimicrobial resistance (AMR). The AMR Action Fund, announced on 9 July, brings together 23 leading biopharma companies, philanthropies, development banks and institutions to support antibiotic R\&D. The aim of this collective effort is to rebuild the biopharma industry's pipeline and bring two to four new antibiotics to patients by 2030 . The fund will launch in the fourth quarter of 2020, when it will begin to invest in, and provide technical support to, smaller biotechs developing innovative antibiotics.

The hope is to avert a looming crisis. Antimicrobial-resistant organisms kill 700,000 people a year, and this number could increase exacerbated by COVID-19related hospitalizations. Although the need for new antibiotics is urgent, market conditions and reimbursement issues have seen big pharma shy away from new antimicrobial development. Small, innovative biotechs have been similarly stung by poor return on investment: Aradigm (recently acquired by Grifols) was forced to seek bankruptcy and so was Achaogen, only a year after its first product was approved by the US Food and Drug Administration. To address such hurdles, the fund will work with the World Health Organization (WHO) and development banks to help governments make the necessary policy reforms. The AMR Action Fund was conceived by the International Federation of Pharmaceutical Manufacturers \& Associations, the Biopharmaceutical CEOs Roundtable and biopharma companies, in collaboration with the WHO, the European Investment Bank and the Wellcome Trust. The collective response was ignited by the WHO's call to action this January when it highlighted the industry's weak pipeline and lack of innovation, despite the growing societal need. Other funds focused on early-stage antimicrobial development are the Combating Antibiotic-Resistant Bacteria Biopharmaceutical Accelerator (CARB-X) and the Denmark-based Novo Holdings' REPAIR Impact Fund. However, experts say these initiatives are only buying time; what is truly needed is for governments to come up with reimbursement reforms and business incentives for a sustainable market for antimicrobials.

Published online: 5 August 2020 https://doi.org/10.1038/s41587-020-0639-4 